\title{
Revealing Phenomena of Heat Energy, Levity, Gravity and Photons Characteristic Current to Light on Dealing Matter to Sub-Atom
}

\author{
Mubarak Ali \\ Department of Physics, COMSATS Institute of Information Technology, \\ 45550 Islamabad, PAKISTAN \\ mubarak74@comsats.edu.pk or mubarak74@mail.com, Ph. +92-51-90495406
}

\begin{abstract}
Technology is in the way to reaching in its climax but the basic understanding of science in many phenomena is still awaited. Scientific research reveals strong analogy between electron and photon. Atoms that execute suitable electronic transitions, on absorbing heat energy at shunt level, excite their electrons. De-excitation of an electron under the gravitational force of its nucleus, where inertia is involved, results depicting energy in the shape like Gaussian distribution. The wavelength of photon remains in inter-state electron's gap where the source of generating energy in wave-like fashion is due to electronic transitions under confined electrondynamics; energy configures under electron's trajectory in the excitation period is due to inertialevitation-inertia behaviours while energy configures under electron's trajectory in the deexcitation period is due to inertia-gravitation-inertia behaviours. Silicon atom is a model system of it. Uninterrupted confined inter-state electron-dynamics results into configure energy in a wave-like fashion that can travel immeasurable length and on interruption from the point of generation, it becomes a photon. Such photons increase wavelength on decreasing energy while travelling through inherently built gap of splitted inert gas atoms where they give light (glow) on reaching wavelength in the visible range. Here, I discuss that heat energy is due to merged photons, photons characteristic current are due to photons having wavelength in interstate electron's gap and light is due to photons, following the wavelength in the visible range. Force of repulsion or attraction in certain materials engages the phenomenon of levitism or gravitism where inertia is exempted. All structural motifs and dynamics are subjected to characteristic photons as long as atoms are under neutral behavior of field force. A structural design delivers straight-forward application on dealing photons at different wavelengths. Here, materials science explores matter at electronic level while absorbing heat energy and generating photon energy. Thus, devise science to describe.
\end{abstract}

Keywords: Heat energy and photon energy; Materials Science; Nanoscale Phenomena; Atomic Scale Phenomena; Sub-Atomic Scale Phenomena

\section{INTRODUCTION:}

Humanity is being benefited by heat energy and light since the existence and electrical phenomena have been studied, since antiquity. Catching fire in various stuffs is a usual phenomenon known since the existence of life. Everyone is taking benefit of those blessings but the understanding behind those phenomena is not clear and then in relation to photon energy coordinating and interacting to various sorts of matter.

A large number of studies are available in the literature, dealing with light-matter interaction, and it has been covered largely under a phenomenon, namely, surface plasmons. Origin of plasmons was explored in some early published reports [1-4]. A plasmon is a quantum of plasma oscillation and represents the collective oscillations of the free electron gas density.

The interaction of light (photon) to matter is recognized in the form of various terminologies, namely, phonon, excitons, and plasmon, etc. Recently published review discusses the light-matter interaction by taking into account the properties of 
polaritons modes in two-dimensional materials, applications at certain range of the spectrum [5]. The concept of excitons (electron-hole pairs) was first proposed by Frenkel [6] and deals with excited state of an atom in a lattice travelling in particle-like fashion without net transfer of charge. Excitons can be formed on absorption of photon by a semiconductor (quantum dot) [7]; a phonon is a collective excitation in a periodic, elastic arrangement of atoms or molecules in condensed matter.

A vast number of studies deal the formation process of tiny-sized particles -structure evolution at nanoscale. The tiny-sized cluster is a simple chemical compound which has a variety of important applications in diversified areas [8]. The unique nature of nanocrystals solicits fabrication of new materials of controlled features [9]. The likely development of nanoparticles technology is an obvious long-term benefit [10]. With the success of assembling colloidal matter in a useful structure, the atoms and molecules will also be treated as materials [11]. The investigation of the dynamics of an individual nanoparticle should be taken as a prime concern prior to going for further solid deliberations [12]. A good understanding on the surface features of nanoparticles will lead the development of higher order materials [13]. Tiny-sized clusters possess molecular-like electronic structures and non-fcc geometric structures [14]. Chemical properties of gold nanoparticles change with size [15]. A variety of strategies to process materials at different length scales have been introduced and discussed in the literature by keeping in view the particular emphasis on their shape and size. From another perspective, research in the field of optics, catalysis, photonics and light is also on the way growing rapidly.

It has been suggested that localized dynamics of the process is one of the cause that helps to evolve the structure of tiny-sized particles in gold [16-20], silver [20], and carbon $[21,22]$. Under the process of synergy or impinging electron streams from the regular external source, electron states of atoms are diffused as per available room while they remained embedded in the tiny particles [16]. A tiny-sized particle where it doesn't have certain geometric structure, it goes for packing under non-uniform drive [17-20]. The atoms of made tiny-sized particles reveal various mechanisms of diffused electron states depending on the process of synergy and external source of impinging electron streams [16]. However, where impinging of electron streams is regular from the connected external source, the underlying atoms of tiny particles reveal orientationalbased diffusion of electron states [16]. The orientational-based diffusion of electron states of atoms while occupying tiny-sized particles may be uniform under the process of synergy $[20,23]$. When the diffusion of electron states of an atom is orientational based, we say stretching of atoms or elongation of their made tiny-sized particles, one-dimensional, and when it is non-orientational based, we say deformation of atoms of their tiny-sized particles as discussed largely [16-24].

Sir Isaac Newton explained the gravity called Newtonian Physics. There is no such concept of anti-gravity in the Theory of General Relativity as explained by Sir Albert Einstein. Bohr proposed that electrons move around the nucleus in allocated orbits where they have fixed energy as long as atom is in ground state. Therefore, the levitation behavior existing with full clarity at electronic level remains beyond the understandings and the concept of band gap, valence band and conduction band dominated along with shells and orbital configurations of electrons kept away to consider and thought on 'force energy' directed phenomena safeguarding the smallest entity of matter (electron) around the nucleus. The more efforts were remained in exploring technological advances and breakthroughs and the efforts put forth toward basic and fundamental sciences along with continuity in the explored ones remained in less focus.

In this work, it has been discussed the heat energy and photon energy, and then how matter at electron scale utilized the heat energy and generated the photon energy. On interrupting a wave generating at point of 
source under confined electron-dynamics executing by a suitable atom, it resulted into long length photon called overt photon, on coordination or interaction to appropriate medium, either placing parallel to or perpendicular to and in the case where not diffracted, transforms into merged photons or squeezed photons, respectively, following by transformation into heat energy and where unit photon merged or squeezed, it results into heat energy called shunt energy. Electrons of all suitable atoms excite under that heat energy called shunt energy on entering or on absorption to those atoms. Whereas, those atoms do not execute electronic transitions, split under the application of photonic current following by increasing wavelength in the visible range on entering through gap made by splitted atoms. Here, charisma of an atom capable to execute electronic transitions under controlled electron-dynamics is discussed while restricting to elastically-driven electronic state behavior. The analogy between electron and photon is explained by taking silicon atom as a model system. This study purely determines the heat energy and the photon energy at the instant of dealing matter at electronic level where role of the field force is being exposed.

\section{RESULTS AND DISCUSSION:}

Under adequate field of photonic current, inert gas atoms split into electron streams and nucleus. On impingement, these electron streams transferred kinetic energy to underlying atoms suitable to evolve structure (tiny particles). Atoms of electronic transitions remained positioned within tiny-sized particle while impinging electrons. The impinged electrons are ejected from the inert gas atoms on split under the application of photonic current [24]. Regardless of that inert gas atoms do not execute electronic transitions they splitted under forced energy of photons characteristic current, that's why, forced out electrons are ejected in the form of streams. This is not only in the case of inert gas atoms but in hydrogen atoms and other suitable substances known in plasma forming elements. On splitting of atoms of substances known to form plasma, electrons are transferred to underlying atoms evolve structure, for example, gold, silver, carbon, etc., as a result, electron states of their atoms are diffused in the orientation of impingement. Such atoms deform or stretch depending on the mode of impinging electron streams (also under the process of synergy) and high resolution transmission microscope images of their tiny-sized particles reveal such behaviours. When all atoms of monolayer two-dimensional structure stretched onedimensionally at electron-solution interface, their electron states diffused orientationally [16]. On travelling (or on placing) photons of hard X-rays at photon-solution interface, their suitable elongated electronic structure modified into smooth elements under the force of their wrapping energy [23]. The mechanisms of deformation and stretching of single atom positioned in tiny-sized particle along with modification into smooth element under the placement of hard X-rays photons are shown in Figure 1 (a)-(f) Photons of hard $X$-rays involve wavelength in $0.10 \mathrm{~nm}$ to 0.20 $\mathrm{nm}$ aligning the stretched electron states around nucleus is possible under them, as they also possess adequate level of energy where melting of such electronic structure can be avoided. Previous studies reveal smooth elements made under the application of such wavelength energy photons [16-23].

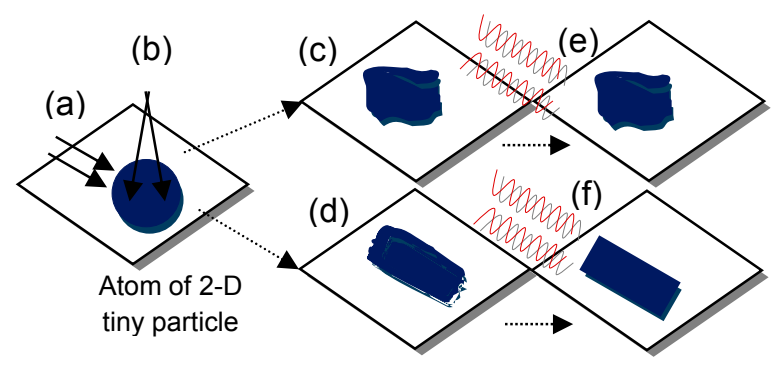

Figure 1: Atom of monolayer two-dimensional tiny particle deals (a) impinging electron streams at fixed angle, (b) impinging electron streams at different angle, (c) deformed atom, (d) stretched atom, (e) travelling (placing) photons of hard $X$ rays didn't modified electronic structure of deformed atom into smooth element and (f) travelling (placing) photons of hard X-rays modify 
electronic structure of stretched atom into smooth element.

In both cases, where either impinging of regular electron streams are not for fixed period to underlying atoms of monolayer tiny particle or the structure of monolayer tiny particle is not in two-dimension, the diffusion of electron states can be non-orientationally based. Their electronic structures do not modify into smooth elements on travelling (placing) photons of hard X-rays and same is the case while packing of such geometric tiny particles into large-sized particles as discussed elsewhere in the case of gold lattice [17-20]. The same is the case in silver lattice [20]. Additionally, the same behaviours of atomic deformation and stretching were observed in 'tiny grains carbon thin films' [21] where elongated one-dimensionally modified into smooth elements while placing (travelling) hard X-rays photons, and some of the discussion is also given elsewhere [22]. Again, geometric monolayer tiny particle was chosen to explain the mechanism of its formation along with stretching and modification into smooth elements [23].

Diffusion process of grains and atoms in various materials under steady state has been explained by the Fick's laws. But the diffusion of electron states of atoms remained unexplained as electrons transferred the forced energy to the ones having capability to stretch or deform on split of certain nature atoms (inert gas atoms) [16]. Marconnet et al. [25] reviewed in details the heat conduction phenomena in carbon nanotubes and related nanostructured films.

The heat energy is not in the form of photon shape-like Gaussian distribution but in the compressed form of Gaussian distribution like shape where they further converted into tits and bits called phonons [26] and they are being absorbed by the suitable material. On the other hand, a photon propagates in interstate electrons' gap of atoms of suitable wire as it is related to the shaped energy having force in any length as long as behaving undependably on the point where generated. Where the configuring energy is dependable on the point of source generating it, it is called a wave as shown in (1) of Figure 2.

The long length photons are called overt photons as shown in Figure 2. Merged photons or squeezed photons are the subset of overt photon in a manner that they resulted on splitting while coordinating or interacting overt photon to the suitable medium, such as, while placing at air-solution interface, either horizontally or perpendicularly. An overt photon involves several nodes and antinodes resulted on termination of a travelling wave at the point of generation in a suitable atom as shown in (1) of Figure 2. Under appropriate coinciding to a medium/surface, an overt photon transformed into merged photons (7) or squeezed photons (8). When a photon having a perfect shape-like Gaussian distribution, which is a unit photon (2), it forms a merged photon under the compression of surrounding medium (3) when placed horizontally (II) with respect to the coordinating or interacting medium as in (7). When a photon having a perfect shape-like Gaussian distribution, which is also a unit photon, it forms a squeezed photon under the compression of surrounding medium (4) when placed vertically $(\perp)$ with respect to the coordinating or interacting medium as in (8). A merged photon or squeezed photon goes into tits bits when it breaks into parts under the coordinated (interacted) medium as in (5) and (6), respectively. However, due to the style of interaction or coordination, the resulted squeezed photons from the over photon may already be related to the heat energies. Thus, they don't require squeezing or compression to use them as heat energy. Therefore, such squeezed photon can be directly be used as shunt energy.

Due to friction, overt photons transformed into merged photons or squeezed photons following by their further division into tits and bits. Now, more likely, on coordinating medium an overt photon mainly transformed into merged photons, while on interacting medium, an overt photon mainly transformed into squeezed photons. An overt photon is the multiple of photon shape-like Gaussian distribution. Thus, an overt photon merges 
into 'merged photons' while placing (travelling) parallel (horizontally) to the medium as shown in Figure 2, whereas, an overt photon squeezes into 'squeezed photons' while placing (travelling) perpendicular (vertically) to the medium as shown in Figure 2. Thus, the difference in formation of merged photon and squeezed photon is the different process of collapsing the overt photon.

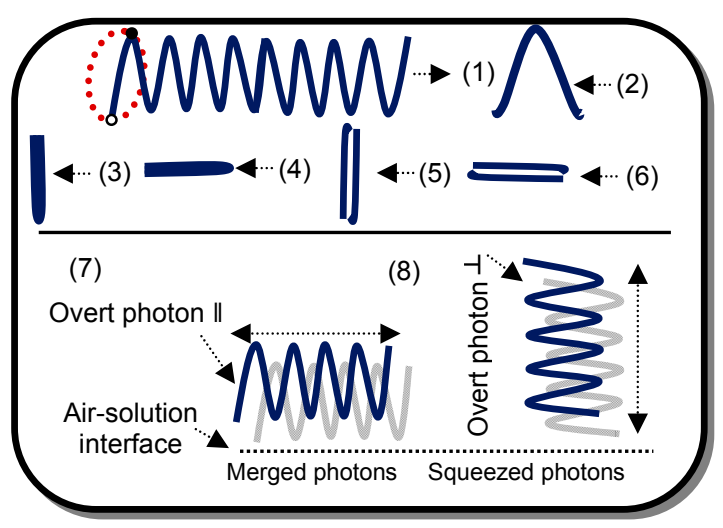

Figure 2: (1) Energy travels in a wave-like fashion configured under the trajectory of an electron, (2) photon shape-like Normal distribution -called a unit photon, on merging, (3) a merged photon, and on squeezing, (4) a squeezed photon (heat energy of both is related to shunt energy), (5) a merged photon and (6) a squeezed photon transformed into tits and bits (the smallest entity of heat energy), overt photon on placing or travelling (7) horizontally (II) or (8) vertically $(\perp)$ at airsolution interface resulted into merged photons or squeezed photons, respectively.

The suitable atom absorbed heat energy is the shunt energy resulted on compression of photon shape-like Gaussian distribution and under the application of that energy an electron goes to excited state, giving out configured energy again shape-like Gaussian distribution on de-excitation, which is also called unit photon and related to 'force energy'. It infers that a merged photon or a squeezed photon owns discrete energy but doesn't the one where energy was configured in Gaussian distribution like shape, therefore, they deal heat energy at shunt level, which is the amount of energy required to free an electron from the inherent force of nucleus at the instant of excitation where that electron goes to excited state under the behavior of levitational force available at electronic level, however, that electron comes back under the force of its nucleus to be remained in dedicated energy pocket of electronic gauge, thus, that atom remains at conserved energy. The heat energy at shunt level remains the same for all atoms having the phenomenon of electronic transitions and different number of electrons in an atom result into varying the temperature of materials at atomic scale, nano scale, micro scale and bulk scale and the detailed work on temperature mapping of different materials will be presented in a separate submission.

The energy of merged photons (or squeezed photons) is related to heat energy, which is being transferred to suitable atoms under certain arrangement. Such heat energy can't be absorbed by the inert gas atoms and also not possible to split them in electron streams and nucleus under such heat energy and then they also do not give 'force energy' as they do not possess room for entering that heat energy as electronic gauge of atoms of all inert elements are filled. However, atoms of suitable electronic transitions are capable to absorb (intake) such heat energy at the location of their vacant state in electronic gauge. Entering of heat energy at shunt level results into execute electron's excitation in those atoms. 'Shunt energy' forces the electron from the back side, frees it from the inherent force of the nucleus, which results into excite that electron resulting into occupy unfilled higher state by configuring energy in the integral sign, when electron comes back under the inherent force of nucleus while deexciting due to gravitation behavior resulting into configure energy shape-like opposite to integral sign, thus, the overall energy configured under the trajectory of electron has the shape like Gaussian distribution, which is a ' force energy' -also called unit photon and this is due to one cycle electron's excitation.

When heat energy of merged photons (or squeezed photons) coordinates to atom like silicon, providing shunt energy to its electron, it is being excited to higher state. As observed 
in solar panel at $45^{\circ}$ angle (approx.) with respect to plane oriented to the south will result into an average maximum power generated throughout the year where configured silicon atoms not only absorbed the heat energy of made merged photons or squeezed photons but also dealt the direct absorption of such photons resulting into bonus power. Therefore, solar panels based on silicon atoms, the probability of electronic excitation becomes higher and systematic resulting into long length photons involving greater energy, thus, an effective generation of current. Electron-dynamics are restricted within inter-state distance (gap) in atoms having the phenomenon of electronic transitions as discussed elsewhere [26]. The cycles of excitation and de-excitation of electrons in silicon atoms are at fast rate and non-stop for longer period. On titling silicon solar panel at certain orientation with respect to base results into varying the efficiency, which is quite effective in the peak hours of sunlight.

In excitation of an electron from the inner state to higher state, the energy configured under the trajectory of electron was mainly due to levitation behavior, when electron was de-excited under its free fall, the energy configured under the trajectory of electron was mainly due to gravitation behavior. That electron included the behaviours of inertia at the start, in the between of levitation and gravitation behaviours, and at the end as well, thus, overall, configured energy is in the shape like Gaussian distribution, which is the 'force energy'. This is a complete cycle of electron, which results into frame energy in shape-like Normal distribution. The same amount of shunt energy is being involved to free that electron again for re-exciting. Continuous phenomenon of excitation and de-excitation of electron within inter-state distance results into an unterminated wave of wavelength equal to distance of inter-state electron's gap. As clarified in Figure 3 showing excitation of electron (black color) under shunt energy. The energy configured under the trajectory where electron changed the state was due to inertia while the energy configured under the trajectory in steady-state behaviors of electron was due to levitation and gravitation behaviors. The process of self-returning of same electron to original state after completing 9 cycles shaped the energy like a wave as shown in Figure 3 and the interruption at the point of source generating it, it becomes the overt photon. The same process takes place in many atoms of the lattice which results in generating photonic current along with some additional modification required for a silicon solar cell. The heat energy of merged photons or squeezed photons, which is the shunt energy required to excite the same electron 9 times is shown in Figure 3. De-excited electron will arrive at same point from where it was excited, each time, and due to topology of an atom, thus, configuring energy shape-like Gaussian distribution against each cycle. However, continued supply of shunt energy to that electron in each cycle of de-excitation to re-excite will lengthen the wave. Thus, in various so-called magnetic materials, the force of repulsion and attraction is not due to magnetism but relates to levitism phenomenon and gravitism phenomenon, respectively, where inertia is exempted due to intrinsic nature of those materials, for example, in iron. The force of repulsion is due to levitism phenomenon while force of attraction is due to gravitism phenomenon.

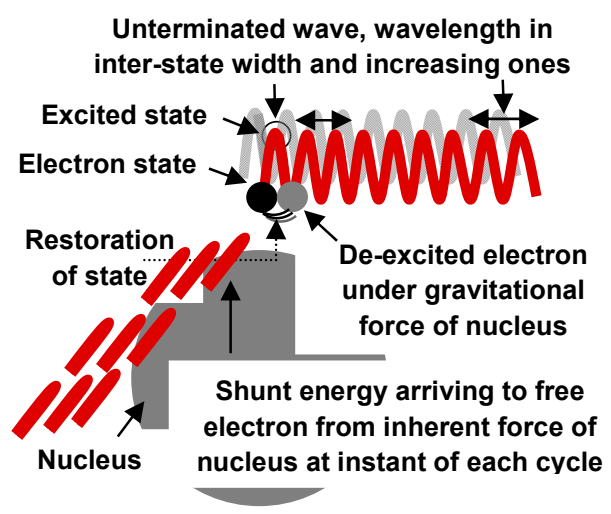

Figure 3: Excitation of electron under shunt energy and de-excitation of electron under the force of nucleus where repeated process of excitation and de-excitation of electron results in 
travel the energy in a wave-like fashion, increasing wavelength with respect to point of generation along with the entering of shunt energy to re-excite electron for each cycle.

A unit photon, which has shape-like Gaussian distribution, on merging, made the shunt energy where the one end (like tip) perfectly fit to back side of the electron, at target, to excite [26]. Therefore, the shunt energy enters into the atom by placing at the backside of the electron located in the lower state resulting into free it from the inherent force of the nucleus, thus, it goes to excited state where energy is configured under made trajectory due to inertia following by levity and then again due to inertia. The overall shape of configured energy is like integral symbol $(\delta)$. At higher state, electron intended to relax for an instant but changed state of rest to state of motion where energy is configured under made trajectory due to inertia following by gravity and then again due to inertia where overall shape of the configured energy is in opposite shape of integral symbol. The tensing of excited electron at higher state and restoring back its state is due to gravitational force of the nucleus acting on it. So, the energy configured in the shape of two integration symbols, opposite to each other, remained connected under the path of electron resulted into give the overall shape of configured energy like Gaussian distribution as shown in Figure 4.

The energy configured along the path when an electron is going to excite under the disturbance of its state of rest, it is due to inertia of the electron, as it is reaching in the state of motion to jump into higher state - (1) to (2) in Figure 4 . In the time of exciting the electron, the energy configured under the trajectory is due to steady-state levitation behavior - between (2) and (3) in Figure 4. On turning the electron to go into higher state, the energy configured is due to inertia of the electron - (3) to mid-position of the curve in Figure 4. When the electron is turned into deexcitation, instead of relaxing at point of higher state, energy configures due to inertia of the electron - mid-position of the curve to (4) in Figure 4. In the course of de-exciting the electron, the energy configured under its trajectory is due to steady-state gravitation behavior - between (4) and (5) in Figure 4. The energy configured on reaching to ground position of electron is again under the inertia as it changed the state from motion to rest (5) to (6) in Figure 4. An equal amount of inertia of electron involved since, to start motion of electron from rest or to come at rest from its motion. Thus, without any breakings, those portions of energy configured under confined electron-dynamics giving out 'force energy', which is called 'unit photon'. On squeezing/compression the arms of unit photon, its 'force energy' is converted into heat energy, which is equal to shunt energy; an energy required to make an electron free from the inherent force of the nucleus.

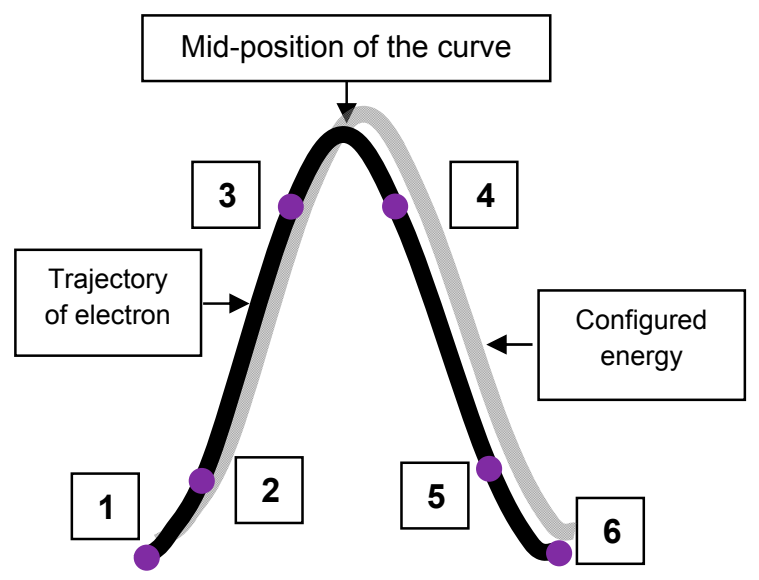

Figure 4: An electron showing configured energy shape like Gaussian distribution under trajectory front side (dark color) shows trajectory followed by the electron while exciting from original filled state to higher vacant state and rear side (grey color) shows configured 'force energy' under the trajectory of electron-dynamics.

In Figure 5, inert gas atoms (argon) are splitted under the photonic current due to having no vacant state of electron in their electronic gauge. Inert gas atoms behave inert because they neither absorb heat energy at shunt level (or at any other level) nor they deform/stretch under the impingement of electron streams and the process of synergy. But they are self utilized to impinge or strike purposes, on split. The capability to absorb 
heat energy from surrounding is related to the emptiness of valence band of an atom, which is not in the case of inert gas atoms. Nevertheless, splitting of atoms switch the wavelength of photons characteristic current to increasing wavelength of photons under their made gap while splitting into electron streams and nucleus as discussed elsewhere [24]. The phenomenon can be observed in Figure 5 where photons characteristic current splitted argon atoms under the required 'force energy', on entering through the gap, made on separation of electron streams and nucleus, increase wavelength where while reaching in the visible range reveal the light (glow).

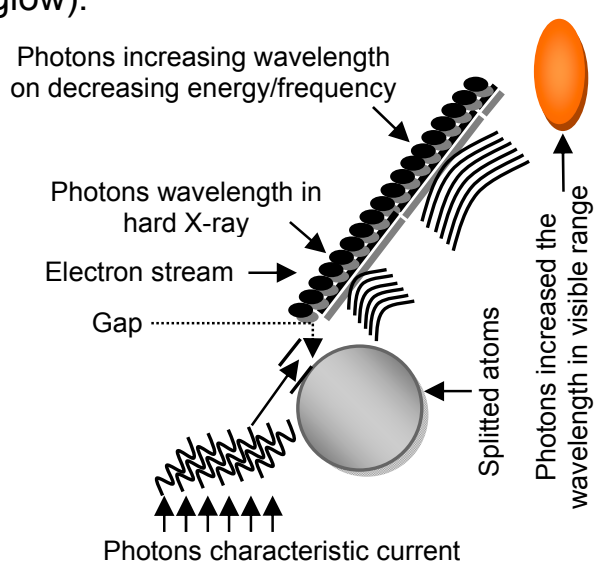

Figure 5: Splitting of argon atoms into electron streams and nucleus where through inherently built gap switch photons of current to increasing wavelength photons.

When a material of certain designed is introduced to deal photonic current, for example, connecting tungsten filament of bulb between so-called negative terminal (where current is propagated) and positive terminal (where surplus current is propagated) result into light as the coil setup is under maintained vacuum and in the case of open air setup as for so-called electric heater where photonic current is mainly converted into heat energy. The same is the case in other household appliances (LCD, tube light, TV, Radio, microwave oven, etc.) and according to builtin features of the certain appliance, utilization of photonic current is operated at different level. When a device breakups, the propagation of photonic current is also halted where positive terminal is no longer remained effective and it is involved to utilize excess photonic current into next appliance connected in series or to propagate energy back to grid.

An electron has mass and it is diffused under the deformation or stretching process of certain nature atoms. In the case of photon, it doesn't have mass and it involves only discrete energy, which is being forced by the design of its shape. In this context, overt photons neither diffused nor impinged and they even don't strike, they either propagate or travel where their energy is being absorbed by the coordinated (or interacted) medium. The nature of coordinated photon itself signified as the energy reveals different behavior; diffract, propagate, absorb, etc. Photons wavelength in visible range enable resolution of the image down to $0.2 \mathrm{~mm}$. Photons energy in the wavelength of $\mathrm{X}$-rays propagate through human body and identify the sign of fracture. Photons characteristic current propagate in suitable wire, thus, they are photonic current. Different cameras and devices manipulate the image on screening diffracted photons at the surface of an object.

When it is said that an electron is spoiled it doesn't mean that the atom of that electron is ionized but it means that the electron occupying atom is no more under elasticallydriven behavior and atom enters in the zone where electron is dealing plastically-driven behavior, thus, such atoms either deform or stretch but do not ionize [24].

In various 'tiny grains carbon thin films' field emission applications are due to formation of smooth elements on the modification of tiny grains having graphitic structure [21]. Those tiny grains of carbon thin films modified electronic structures into smooth elements are the source of enhanced field emission and display panel applications. As photonic current propagates through modified smooth elements under enhanced field, thus, they are utilized straight-forwardly for field emission applications or display panels. The performance of such 'tiny grains carbon thin films' should be notified in terms of formation 
of maximum smooth elements of tiny grains followed by the nature of their structural connections and such films can give phenomenal controlled features. The same approach should be considered in studying phenomena like surface enhanced Raman scattering or localized surface Raman spectroscopy of nanoparticles [27].

Distribution of heat energy to disordered structure is in non-uniform manner. More heat is resulted in structures where atoms are randomly distributed as photons characteristic current dissipate heat energy within such structure by mingling to each other, crossing, overlapping, thus, raise the temperature (heat) of the structure. Collapsed photons in tits and bits can again transform into merged photons or squeezed photons, thus, working as the heat energy for suitable atoms. Similarly, when photons of different energy disrupt the medium (overt photons), they dissipate heat energy in the form of merged photons or squeezed photons. Matter assigns different roles to photons on their interaction or coordination, converting their one form of energy to another and depending on the structure and topology. In Bragg's diffraction, amorphous materials don't reveal any specific structure under the interaction of photons wavelength in X-rays. Photons, on altering shape along with 'force energy' work as heat energy through which atoms of suitable elements excite electrons generating photons characteristic current, which propagate in metallic wires while working as a photonic current. In some cases, photons characteristic current are utilized to split the matter like inert gas atoms and their electrons streams are being utilized to deform or stretch atoms of electronic transitions.

The set modalities of all sorts of photons depend on the origin of their generation establishing roles set by the manufacturer or expert under the coordination or interaction to characteristics matters. In this context, structural design is crucial in targeting their specific application and many studies are now targeting and exploring structure either standalone or in relation to other fields of science [28-37]. In materials with two- dimensional structure uniform photon couplings prevail $[23,26]$ while in threedimensional materials non-uniform photon couplings prevail [26]. To measure temperature of such materials and in their selective sizes is the integral part to understand science of their different behaviours and some of the studies shed light [38-40].

On moving optical tweezers in real-time control system, tunable arbitrary geometries of cold neutral atoms for quantum engineering are prepared [41] and regular arrays of individual controlled cold atoms as well are prepared [42]. On one side, the scope of inert gases is declining due to explanation that these are not the ones forming fourth state of matter known as plasma and they only pass the photons into the gap, on the splitting, to exceed their wavelength from shorter to longer where they reveal the light (glow); on reaching in visible range. Some of its detail is also given elsewhere [24]. On the other side, these recently published studies [41, 42] are opening the new avenues of research as well. Such tunable arbitrary geometries and regular arrays of atoms, on further tuning, may become the future candidates for hiding selective text of a document and many other interesting applications. As atoms of inert gases are the serving candidates as either they are exceeding wavelength of photons characteristic current into higher ones or their electron streams, on split, impinging to underlying matter of suitable atoms resulting into their stretching or deformation as per available room in their electronic gauge. Definitely, before serving they own expertise and need to be explored. A recent study explained the role of van der Waals interactions in the case of isolated atom [43]; they arise from induced dipoles, which can be attained when fluctuations of charge density are in wavelike nature [44]. Our several investigations discuss that structural motifs are owing to dynamics of the process arisen by characteristics photons and field forces [16-24] where it is proven experimentally in one of the study given elsewhere [18]. However, where the behavior of field forces 
are neutral, the only characteristics photons play role in controlling the structure of suitable material as in the case of graphite and in other suitable two-dimensional structure depending on the process parameters. However, characteristics photons and field force behaviours are at forefront in the design of any sort of material and it is not possible to design even ordinary featured structure without their involvement [18]. All that glitters need not be Au but TiN or ZrN as well [45]. It appears that elements and compounds where atoms execute electronic transitions, they all glitter, and what is needed is to attain shunt energy to excite electron in atoms under their confined dynamics. Materials of those atoms evolved the structure in order of few atoms generating the photons at different wavelength from the point of source. Thus, it becomes difficult to observe the light with naked eye as the resolute power of the image becomes smaller than the ability to view it $(<$ $0.2 \mathrm{~mm}$ ). It is possible to measure temperature of atoms, their nanoscale components and at bulk scale as well without the involvement of thermocouples and introducing various gadgets, theories, etc. What is required, to determine the probability of exciting electrons at shunt energy in the atoms revealing the phenomenon of electronic transitions? Atoms with less number of electrons reveal higher temperature owing to lower probability of exciting electrons under available heat energy. In fact, heat energy of suitable merged photons or squeezed photons transferred to atoms under their self-force in the course of merging or squeezing, which is referred to localized heating of atoms at which electrons excite, on entering, they compress resulting into form shunt energy. In addition to evolution of structure in graphitic phase, amorphous and modified phase of graphite structure (those modified into smooth elements), a 'tiny grains carbon thin films' also reveal diamond, lonsdaleite and graphene phases in Raman spectroscopy as well as in energy loss spectroscopy [21]. Our recent investigations [46] show that besides characteristics photons, a field force, either due to levitation behavior or due to gravitation behavior or due to their mixed behavior involve evolving a variety of structure along with introducing various modifications in the evolved (or evolving) structure; within same material (carbon atoms) and various modified phases are discussed (fullerene, diamond, lonsdaleite and graphene) under the variation of field force behaviors at electronic level. A recent study expressed the silent feature and implication of modified structure at electronic level while using as nanomedicine [47].

\section{CONCLUSIONS:}

Stretching and deformation behaviours of atoms of electronic transitions take place under the impinging/striking of electrons where their electron-dynamics do not remain confine, whereas, internally absorbed heat energy (called shunt energy) by such atoms enables the elastically-driven electronic state behavior where energy is configured in the shape like Gaussian distribution and under the made trajectory of electron during each cycle. Such photon is called unit photon, which is the heat energy at shunt level of an electron at target, on merging (following by compression), or on squeezing. On interrupting the energy configuring in a wave-like fashion at the point of source of generating, it becomes the overt photon, which is a long length photon, when coordinated or interacted to suitable medium, it transforms into either merged photons (on parallel placing to the medium) or squeezed photons (on perpendicular placing to the medium). A merged photon or a squeezed photon under uniform compressing form the basis of heat energy called shunt energy, which is required to excite electron of atoms of all suitable elements, on entering through the unfilled state of electron in the electronic gauge, enables the electron to free from the inherent force of the nucleus resulting into excite it where the energy is configured in shape-like integral symbol, however, that electron doesn't relax in the higher state due to revealing the phenomenon of inertia following by the gravitism and then again 
inertia where the energy is configured in opposite integral symbol. The de-excitation of electron is under the gravitational force of the nucleus. Thus, the energy configured under the trajectory of an electron in each cycle is termed as unit photon shape-like Gaussian distribution and connecting such shaped energy under unterminated cycles resulting into travelling of energy in a wavelike fashion, on interrupting at any stage (at two or more than two cycles of electron's excitation) result into give photon called overt photon, which is again transformed into merged photons or squeezed photons under the appropriate coordination or interaction to the medium. Therefore, heat energy and photon energy work in a cycle where central part is a suitable atom implementing confined electron-dynamics under the absorption of heat energy at shunt level where the continuous process of electron's excitation results in configuring the heat energy into photon energy following by reversing the process, again.

Exciting electrons under confined dynamics while supplying uninterrupted heat energy results into immeasurable length of energy travelling in a wave-like fashion, on interruption called a photon and on collapsing called heat energy. In various materials, structural adjustments to different forms of energy find the way out for certain application, indicating that design of the material manipulates energy for targeted application. Photons characteristic current, when it collectively propagates in suitable wire, it is a photonic current. An electron has strong analogy to photon in a manner that inter-states electron's motion result into configure energy shape-like a wave where at each state of changed motion, inertia is involved.

Structure of certain materials deliver straight-forward application on interacting or coordinating (or both) to heat energy or photon energy, for example, solutions of various metallic colloids and quantum dots, on interacting light, split light into different colors depending on the distance of interstate electron's gap along with size and shape of the nanoparticles/particles and 'tiny grains carbon thin films' enhance field emission characteristic under the increased distance of inter-state electron's gap. Atoms of inert gases and other suitable substances split under photonic current providing gap to photons characteristic current exceed in their wavelength and while reaching in the visible range we observe a glow or light known as plasma. In certain materials, the force of repulsion or attraction is due to levitism or gravitism phenomenon instead of magnetism and incites Coulomb's Law. When inertia is involved depending on the nature of electronic gauge of atom, the resulted behavior of an electron deals it as well, whenever changed the state and responsible for travelling the energy in a wave-like fashion, which propagates in a suitable wire to work as current, thus, inciting electromagnetic spectrum. All suitable atoms of electronic transitions are naturally built-in machines (devices) to utilize heat energy and transform into photon energy and atoms of silicon lattice are the best choice to deliver photon energy in long length, thus, work as photonic current. The matter is nothing without energy and all matters operate under it. An electron is also matter, perhaps, the smallest entity of matter and it is the most valuable when it works within natural confinement (domain).

Clearly, electrons are matter, occupy space, possess mass and impinge (strike) under given energy from external source while they excite/de-excite from internal source under the absorption of shunt energy. Whereas, all types of photons possess energy as well as force but not mass, they use space in propagation (within the gap of source/medium dealing their wavelength) or travelling while dissipating heat (on collapsing shape) where their force is diminished but their energies work, thus, they are not the ones impinging (striking) or exciting/de-exciting electrons.

\section{References}

[1] D. Bohm, D. Pines, A Collective Description of Electron Interactions. I. Magnetic Interactions, Phys. Rev. 1951, 82, 625-634. 
[2] D. Pines, D. Bohm, A Collective Description of Electron Interactions: II. Collective vs Individual Particle Aspects of the Interactions, Phys. Rev. 1952, 85, 338-353.

[3] R. H. Ritchie, Plasma Losses by Fast Electrons in Thin Films, Phys. Rev. 1957, 106, 874-881.

[4] D. Bohm, D. Pines, A Collective Description of Electron Interactions: III. Coulomb Interactions in a Degenerate Electron Gas, Phys. Rev. 1957, 92, 609-625.

[5] T. Low, et al., Polaritons in layered twodimensional materials, Nat. Mater. 2017, 16, 182194.

[6] J. Frenkel, On the Transformation of Light into Heat in Solid. I, Phys. Rev. 1931, 37, 17-44.

[7] O. D. D. Couto Jr. et al., Charge control in InP/(Ga, In)P single quantum dots embedded in Schottky diodes, Phys. Rev. B 2011, 84, 125301-7.

[8] M. Brust, M. Walker, D. Bethell, D. J. Schiffrin, R. Whyman, Synthesis of Thiol-derivatised Gold Nanoparticles in a Two-phase LiquidLiquid System, J. Chem. Soc., Chem. Commun. 1994, 801-802.

[9] R. L. Whetten, J. T. Khoury, M. M. Alvarez, S. Murthy, I. Vezmar, Z. L. Wang, P. W. Stephens, C. L. Cleveland, W. D. Luedtke, U. Landman, Nanocrystal Gold Molecules, Adv. Mater. 1996, 8, 428-433.

[10]M. Brust, C. J. Kiely, Some recent advances in nanostructure preparation from gold and silver particles: a short topical review, Colloids and Surfaces A: Physicochem. Eng. Aspects 2002, 202, 175-186.

[11]S. C. Glotzer, M. J. Solomon, Anisotropy of building blocks and their assembly into complex structures, Nature Mater. 2007, 6, 557-562.

[12] S. Link, M. A. El-Sayed, Shape and size dependence of radiative, nonradiative and photothermal properties of gold nanocrystals, Int. Rev. Phys. Chem. 2000, 19, 409- 453.

[13] C. P. Shaw, D. G. Fernig, R. Lévy, Gold nanoparticles as advanced building blocks for nanoscale self-assembled systems, J. Mater. Chem. 2011, 21, 12181-12187.

[14]Y. Negishi, T. Nakazaki, S. Malola, S. Takano, Y. Niihori, W. Kurashige, S. Yamazoe, T. Tsukuda, H. Häkkinen, A Critical Size for Emergence of Nonbulk Electronic and Geometric Structures in DodecanethiolateProtected Au Clusters, J. Am. Chem. Soc. 2015, 137, 1206-1212.
[15]A. Moscatelli. Gold nanoparticles: Metallic up to a point, Nature Nanotechnol. 2015, DOI:10.1038/nnano.2015.16.

[16](a) M. Ali, I -Nan. Lin, The effect of the Electronic Structure, Phase Transition and Localized Dynamics of Atoms in the formation of Tiny Particles of Gold, http://arXiv.org/abs/1604.07144 (2016).

(b) M. Ali, I -Nan. Lin, The effect of the Electronic Structure, Phase Transition and Localized Dynamics of Atoms in the formation of Tiny Particles of Gold, J. Nanopart. Res. 2017, 19, 15.

[17]M. Ali, I -Nan. Lin, Geometric structure of gold tiny particles at varying precursor concentration and packing of their electronic structures into extended shapes, http://arXiv.org/abs/1604.07508 (2016).

[18]M. Ali, I -N. Lin, Controlling morphologystructure of particles via plastically driven geometric tiny particles and effect of photons on the structures under varying process conditions, http://arxiv.org/abs/1605.04408 (2016).

[19]M. Ali, I -N. Lin, Formation of tiny particles and their extended shapes-origin of physics and chemistry of materials, http://arxiv.org/abs/1605.09123 (2016).

[20]M. Ali, I -N. Lin, Dynamics of colloidal particles formation in processing different precursors-elastically and plastically driven electronic states of atoms in lattice, http://arxiv.org/abs/1605.02296 (2016).

[21] M. Ali, I -Nan. Lin, Phase transitions and critical phenomena of tiny grains thin films synthesized in microwave plasma chemical vapor deposition and origin of v1 peak, http://arXiv.org/abs/1604.07152 (2016).

[22]M. Ali, M. Ürgen, Switching dynamics of morphology-structure in chemically deposited carbon films-a new insight, http://arxiv.org/abs/1605.00943 (2016).

[23] M. Ali, Atomic binding, geometric monolayer tiny particle, atomic deformation and onedimensional http://arxiv.org/abs/1609.08047 (2016).

[24] M. Ali, Atoms deform or stretch, do not ionize, those having electronic transitions are the source of photonic current while inherently making terminals of inert gas atoms switch photonic current into photons, http://arxiv.org/abs/1611.05392 (2016).

[25] A. M. Marconnet, M. A. Panzer, K. E. Goodson, Thermal conduction phenomena in carbon nanotubes and related nanostructured 
materials, Rev. Mod. Phys. 2013, 85, 12951326.

[26]M. Ali, Structure evolution in atoms having phenomenon of electronic transitions, http://arxiv.org/abs/1611.01255(2016).

[27] S. Nie, S. R. Emory, Probing Single Molecules and Single Nanoparticles by SurfaceEnhanced Raman Scattering, Science 1997, 275, 1102-1106.

[28]J. Zhao, L. Yang, Structure Evolutions and Metallic Transitions in $\operatorname{In}_{2} \mathrm{Se}_{3}$ Under High Pressure, J. Phys. Chem. C 2014, 118, 54455452.

[29]V. N. Manoharan, Colloidal matter: Packing, geometry, and entropy, Science 2015, 349, 1253751.

[30]J. Park, et al., 3D structure of individual nanocrystals in solution by electron microscopy, Science 2015, 349, 290-295.

[31]D. Jacobsson, et al., Interface dynamics and crystal phase switching in GaAs nanowires. Nature 2016, 531, 317-322.

[32] T. Tuma, A. Pantazi, M. L. Gallo, E. Eleftheriou, Stochastic phase-change neurons. Nature Nanotech. 2016, 11, 693-699.

[33] J. Rensberg, et al., Active Optical Metasurfaces Based on Defect-Engineered Phase-Transition Materials, Nano Lett. 2016, 16, 1050-1055.

[34] Y. Suzuki, G. Cardone, D. Restrepo, P. D. Zavatteri, T. S. Baker, F. A. Tezcan, Selfassembly of coherently dynamic, auxetic, twodimensional protein crystals, Nature 2016, 533, 369-373.

[35] C. H. J. Evers, J. A. Luiken, P. G. Bolhuis, W. K. Kegel, Self-assembly of microcapsules via colloidal bond hybridization and anisotropy, Nature 2016, 534, 364-368.

[36] I. R. Epstein, B. Xu, Reaction-diffusion processes at the nano- and microscales. Nature Nanotech. 2016, 11, 312-319.

[37]A. Azizi, Spontaneous Formation of Atomically Thin Stripes in Transition Metal Dichalcogenide Monolayers, Nano Lett. 2016, 16, 6982-6987.

[38] M. Mecklenburg, W. A. Hubbard, E. R. White, R. Dhall, S. B. Cronin, S. Aloni, B. C. Regan,
Nanoscale temperature mapping in operating microelectronic devices, Science 2015, 347, 629-632.

[39] L. Ye, D. Hou, X. Zheng, Y. Yan, M. D. Ventra, Local temperatures of strongly-correlated quantum dots out of equilibrium, Phys. Rev. $B$ 2015, 91, 205106-8.

[40]F. Menges, et al., Temperature mapping of operating nanoscale devices by scanning probe thermometry, Nat. Commun. 2016, 7:10874 doi: 10.1038/ncomms10874.

[41](a) D. Barredo, S. de Léséleuc, V. Lienhard, T. Lahaye, A. Browaeys, An atom-by-atom assembler of defect-free arbitrary $2 d$ atomic arrays, https://arxiv.org/abs/1607.03042, (2016)

(b) D. Barredo, S. de Léséleuc, V. Lienhard, T. Lahaye, A. Browaeys, An atom-by-atom assembler of defect-free arbitrary twodimensional atomic arrays, Science 2016, 354, 1021-1023.

[42] (a) M. Endres, et al., Cold Matter Assembled Atom-by-Atom, https://arxiv.org/abs/1607.03044, (2016).

(b) M. Endres, et al., Atom-by-atom assembly of defect-free one-dimensional cold atom arrays, Science 2016, 354, 1024-1027.

[43] S. Kawai, et al., Van der Waals interactions and the limits of isolated atom models at interfaces, Nat. Commun. (2016) DOI: 10.1038/ncomms11559.

[44] A. Ambrosetti, N. Ferri, R. A. DiStasio Jr., A. Tkatchenko, Wavelike charge density fluctuations and van der Waals interactions at the nanoscale, Science 2016, 351, 1171-1176.

[45]A. Boltasseve, V. M. Shalaev, All that glitters need not be gold, Science 2015, 347, 13081310.

[46] M. Ali, Why some atoms are in gaseous state and some in solid state but carbon work on either side (2017). (Submitted for consideration).

[47] M. Ali, Nanoparticles -Photons: Effective or Defective Nanomedicine. Preprints (2017). http://www.preprints.org/manuscript/201703.0 174/. 


\section{Author's biography:}

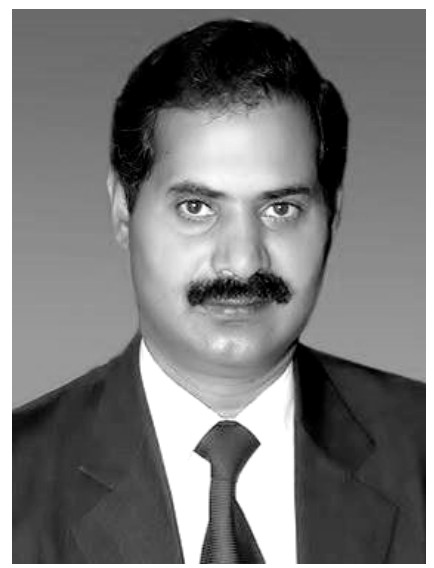

Mubarak Ali graduated from University of the Punjab with B.Sc. (Phys\& Maths) in 1996 and M.Sc. Materials Science with distinction at Bahauddin Zakariya University, Multan, Pakistan (1998); thesis work completed at Quaid-i-Azam University Islamabad. He gained Ph.D. in Mechanical Engineering from Universiti Teknologi Malaysia under the award of Malaysian Technical Cooperation Programme (MTCP;2004-07) and postdoc in advanced surface technologies at Istanbul Technical University under the foreign fellowship of The Scientific and Technological Research Council of Turkey (TÜBITAK; 2010). He completed another postdoc in the field of nanotechnology at Tamkang University Taipei (2013-2014) sponsored by National Science Council now M/o Science and Technology, Taiwan (R.O.C.). Presently, he is working as Assistant Professor on tenure track at COMSATS Institute of Information Technology, Islamabad campus, Pakistan (since May 2008) and prior to that worked as assistant director/deputy director at M/o Science \& Technology (Pakistan Council of Renewable Energy Technologies, Islamabad; 2000-2008). He was invited by Institute for Materials Research (IMR), Tohoku University, Japan to deliver scientific talk on growth of synthetic diamond without seeding treatment and synthesis of tantalum carbide. He gave several scientific talks in various countries. His core area of research includes materials science, condensed-matter physics \& nanotechnology. He was also offered the merit scholarship (for PhD study) by the Government of Pakistan but he couldn't avail. He is author of several articles published in various periodicals (https://scholar.google.com.pk/citations?hl=en\&user=UYjuhDwAAAAJ) and also a book. 